\title{
Le bâtiment monumental de Beaurieux « La Plaine » : bâtiment unique ou évolution terminale du modèle danubien?
}

\section{Caroline Colas}

\section{(2) OpenEdition \\ 1 Journals}

Édition électronique

URL : https://journals.openedition.org/archeopages/481

DOI : 10.4000/archeopages. 481

ISSN : 2269-9872

Éditeur

INRAP - Institut national de recherches archéologiques préventives

Édition imprimée

Date de publication : 1 janvier 2012

Pagination : 100-102

ISSN : 1622-8545

\section{Référence électronique}

Caroline Colas, « Le bâtiment monumental de Beaurieux « La Plaine » : bâtiment unique ou évolution terminale du modèle danubien ? ", Archéopages [En ligne], Hors-série 3 | 2012, mis en ligne le 01 janvier 2012, consulté le 24 février 2023. URL : http://journals.openedition.org/archeopages/481 ; DOI :

https://doi.org/10.4000/archeopages.481 
est paradoxalement bien moins important quà l'étape précédente du Néolithique. Bien souvent, le Néolithique moyen n'est représenté que par quelques fosses isolées renfermant un peu de mobilier.

À la très grande homogénéité de l'architecture danubienne, semble succéder une grande variabilité des architectures qui pourraient également correspondre à la diversification des cultures et à une rupture avec la civilisation danubienne.

Les rares plans de maisons connues pour le début du Néolithique moyen sont très divers, parfois sujets à discussion, et se partagent entre bâtiments de forme circulaires et architectures de plan quadrangulaire. Cependant, ces dernières années, quelques découvertes remarquables sont venues heureusement documenter le début du Néolithique moyen et permettent, au moins partiellement, de retracer l'évolution des l'architecture et de l'habitat à la suite des bâtiments danubiens.

C'est à Pont-sur-Seine, dans l'Yonne, que d'importants ensembles architecturaux datés du Néolithique moyen au Néolithique récent, ont été mis au jour ces toutes dernières années. Au moins quatre structures circulaires ont été repérées et fouillées par Vincent Desbrosse et son équipe. Les architectures ont une superficie d'environ $80 \mathrm{~m}^{2}$ et deux d'entre elles présentent un espace interne cloisonné par une ligne de poteaux. Leur datation se situe aux alentours de 4500 avant notre ère. La fonction de ce type d'architecture n'est pas encore déterminée. Est-elle religieuse, cérémonielle, collective ou s'agit-il d'une habitation?

À Vivoin «Le Parc » (Sarthe), un bâtiment sub-trapézoïdal long d'une vingtaine de mètres, analogue aux architectures danubiennes, mais à l'orientation sensiblement différente, (nord-nordouest), a été récemment mis au jour (Ghesquière, Marcigny, 2003). Il est formé de 42 trous de poteau et l'espace interne est subdivisé par trois tierces : les 2 premières sont renforcées au niveau de l'axe par 3 poteaux qui ont pu soit consolider la charpente, soit soutenir une plateforme en élévation. Les fosses latérales sont absentes, mais plusieurs foyers lui sont associés. Le bâtiment et les structures annexes ont livré un mobilier céramique et lithique assez abondant.

À Beaurieux, « La Plaine » (Aisne), le très vaste bâtiment de forme trapézoïdale est inédit et est également daté de la première étape du Néolithique moyen [cf. encadré ci-contre].

La fonction de toutes ces structures reste problématique mais, selon toute vraisemblance, à une morphologie correspondent une fonction et un rôle spécifique.

[...]
Le bâtiment monumental de Beaurieux «LaPlaine»: bâtiment uniqueou évolution terminale du modèle danubien?

Caroline Colas
Inrap

e bâtiment monumental de Beaurieux

«La Plaine» se trouve au sein d'une nécropole monumentale qui comprend deux à trois monuments funéraires appartenant à la culture du Cerny (4.700-4.300 av. notre ère), un monument funéraire du Michelsberg ancien (4700-4300 av. notre ère) ainsi que quatre sépultures allant du Michelsberg ancien au Michelsberg récent (4 000-3500 av. notre ère). Ce bâtiment a déjà fait l'objet d'une présentation, c'est pourquoi nous n'en rappellerons ici que les principaux éléments (Colas et al., 2008 ; Colas et al., 2010). Sa longueur minimale était de $80 \mathrm{~m}$, mais les dimensions initiales de l'édifice sont inconnues du fait de la destruction ancienne de sa partie ouest. Orienté est-ouest, cet édifice comprend 43 trous de poteaux organisés en onze tierces et 10 fosses pour la façade avant. Sa façade est (20 $\mathrm{m}$ de large), opposée à la façade ouest ( $6 \mathrm{~m}$ de large), lui donne une forme trapéziforme prononcée [fig. 1]. Le module des trous de fondation à poteau unique varie de 1,50 à $2 \mathrm{~m}$. Les fosses de la rangée centrale et de la façade occidentale sont les plus profondes [fig. 2 et 3]. L'ancrage des trous de poteaux de la façade avant s'avère, en revanche, moindre. L'organisation d'ensemble, les comblements et les dynamiques de remplissage similaires ont permis de rejeter l'hypothèse d'une construction en plusieurs parties; seul un poteau indique l'existence d'une reprise.

Comme c'est souvent le cas pour les bâtiments, les comblements des structures de fondation renfermaient peu de mobilier : les tessons (matériaux et décors) évoquent la culture du Cerny et l'existence de plusieurs fragments d'os nous a permis d'avoir recours aux datations isotopiques (carbone 14, méthode AMS). L'intervalle de $4800 a ̀ 4400 \mathrm{av}$. notre ère fourni par ces dernières a confirmé la datation obtenue par le mobilier sans apporter davantage de précision, puisqu'il couvre l'intégralité de la fourchette chronologique attribuée au Cerny.

Deux « épandages », situés de part et d'autre de l'édifice (l'un à une dizaine de mètres au sud et l'autre à une cinquantaine au nord) ont également livré un ensemble homogène de mobilier daté du Cerny éponyme, tandis qu'aucune autre période n'est représentée à proximité de ce bâtiment.

Les données architecturales pour le Néolithique moyen I sont particulièrement ténues. Les rares plans de maisons connus sont très variables : on relève des bâtiments rectangulaires orientés nordsud par exempleà Vivoin dans la Sarthe (Marcigny, Ghesquière, 1999), des maisons trapéziformes estouest à Herblay dans le Val d'Oise (Valais, 1995), ainsi que des maisons rondes, notamment dans le centre de la France (Verjux, 2007) en rupture totale avec tout ce qui précède. L'attribution des trois bâtiments voisins de Berry-au-Bac « Le Vieux Tordoir », dans l'Aisne, au Cerny, ne semble pas plus 
pertinente, compte tenu des données nouvelles sur l'habitat du Néolithique final (Dubouloz et al., 1996). Bien qu'il semble proche des édifices danubiens plus anciens (Coudat, 1998) - forme trapézoïdale, orientation, tierces) et qu'il pourrait apparaître comme une évolution ultime de ces derniers, il s'en démarque par ses dimensions, par l'absence du couloir (deux tierces plus rapprochées) et des fosses latérales qui servaient à extraire les matériaux nécessaires à la fabrication des murs. L'absence des fosses de construction découle peut-être de l'absence de murs. En effet, aucune preuve archéologique ne permet, aujourd'hui, d'envisager leur existence. La très bonne conservation des trous de poteaux et l'existence des deux couches voisines - vraisemblables niveaux de sols - démontrent que l'érosion n'a guère de chance d'être responsable de la disparition des poteaux de parois ou de sablières basses. En l'absence de ces murs, il faut donc imaginer une construction ouverte. Le gabarit et la profondeur spectaculaires des trous de poteaux, notamment de toute la rangée centrale, sont en adéquation avec les dimensions générales et la distance importante de certaines travées ; ces éléments autorisent à imaginer l'existence d'un toit à double pente, semblable à ceux qui devaient recouvrir les maisons d'habitations antérieures. L'allure générale de l'édifice serait proche de celle des halles de marché médiévales.La longueur du bâtiment de Beaurieux fait aussi exception. Les seuls bâtiments de même dimension sont les grandes maisons post-rubanés contemporaines d'Allemagne ou les grandes maisons du groupe polonais de Brzesc Kujawski chronologiquement un peu plus récentes (Hampel, 1989). Mais ces constructions ont toujours des poteaux de parois ou une sablière basse, certaines n'ont d'ailleurs plus que les sablières basses, et leur organisation interne est très déstructurée.

On peut imaginer que l'interprétation très « libre » du modèle danubien provient de son implantation auprès d'une nécropole monumentale. Il est probable que le bâtiment de Beaurieux n'appartenait pas à la sphère domestique, mais plutôt à la sphère religieuse.

COLAS C. BAILLIEU M., NAZE Y., 2008 : « Un bâtiment monumental Cerny à Beaurieux « La Plaine » (Aisne) », Internéo, 7, p. 59-69.

Colas C. (dir), Allard P., Chartier M., Hachem L., Maigrot Y., 2010 : Beaurieux (Aisne) La Plaine, Un « bâtiment monumental » exceptionnel, Rapport final d'opération, Inrap, SRA Picardie, 92 p., 68 fig

COUDART A., 1998 : Architecture et société néolithique. L'unité et la variance de la maison danubienne, DAF, 67, Paris, 240 p., 194 fig.

Dubouloz J., Farruggia J.-P., Robert B., 1996 : « Bâtiments néolithiques non-rubanés à Berry-au-Bac "Le Vieux Tordoir", Aisne : présentation préliminaire », Internéo, p. 51-69.

Hampel A., 1989: Die Hausentwicklung im Mittelneolithikum Zntraleuropa, Universitätsforchungen zur Prähistorischen Archäologie 1, Bonn, Rudolf Habelt, 360 p, 68 fig.

MARCigny C., GHesquiere E., 1999 : « Un bâtiment néolithique moyen I dans la Sarthe », Bulletin de la Société Préhistorique française, t. 96, fasc. 2, p. 264-266.

VALAIS A., 1995: « Deux bâtiments atypiques associés à du matériel Cerny (Herblay, Val d'Oise) ", in Billard C. (DIR), Evreux, Actes du 2oe colloque sur le Néolithique, 1993, Revue Archéologique de l'Ouest ( $7^{\mathrm{e}}$ supplément), p. 57-63.

VERJUX C., 2007 : « Les bâtiments circulaires du Néolithique moyen dans le Bassin parisien », in Agogue O., Leroy D., Verjux C. (DIR) Camps, enceintes et structures d'habitat néolithiques en France septentrionale, Actes du 24e Colloque interrégional sur le Néolithique, Orléans, 19-21 novembre 1999, Revue Archéologique du Centre de la France (2 $7^{\mathrm{e}}$ supplément), p. 209-216.
[...]

L'éclosion d'ouvrages monumentaux :

les enceintes. La société néolithique connaît d'importantes transformations que l'on peut notamment identifier avec l'éclosion d'ouvrages monumentaux, requérant une main d'œuvre nombreuse et une organisation collective ad hoc, notamment pour aménager des enceintes visibles qui marquent le paysage. Il s'agit en effet d'abattre des centaines d'arbres, de les déplacer et ériger les fûts en palissade. Le principe architectural de ces structures consiste à creuser un ou plusieurs fossés continus ou discontinus et une palissade de bois interne est édifiée sur un talus aménagé en utilisant les matériaux extraits des fossés. Les entrées correspondent à l'interruption du fossé et de la palissade, mais peuvent être également conçues en chicane ou en couloir. Ces travaux se sont probablement déroulés collectivement, sous la conduite d'un chef. Leurs fonctions sont encore sujettes à discussion: habitat? Déroulement de cérémonies? Parcage des troupeaux? Les enceintes ont pu également jouer un rôle défensif ou protecteur, puisque la néolithisation étant achevée, les groupes néolithiques s'approprient désormais pleinement un territoire ou un terroir.

Les enceintes sont connues dès la fin du Néolithique ancien, mais se développent surtout dans la deuxième partie du Néolithique moyen. Mais, si ces importantes structures sont relativement bien attestées pour ces périodes, peu d'entre elles avaient été intégralement décapées et fouillées jusqu'au développement des travaux d'archéologie préventive. De même, les vestiges découverts dans l'espace interne de ces constructions étaient généralement erratiques et ne paraissaient pas constituer des habitats pérennes. En Auvergne, toute une série d'occupations du Néolithique moyen II, trouvées dans des contextes géomorphologiques divers, ont été mises au jour [cf. article p 117]. Elles combinent enclos, bâtiments rectangulaires, parfois à deux nefs sur tranchées de fondation et poteaux centraux porteurs, cabanes, aires d'activités spécialisées, structures foyères, formant un tissu de hameaux habités entre 3900 et 3500 av. notre ère, et attestant d'une augmentation importante de la population. À Cuiry-les-Chaudardes [cf. encadré p 103], l'occupation Michelsberg est représentée par quatre bâtiments d'assez petit module dont l'espace interne n'est pas subdivisé.

Le Néolithique moyen correspond clairement à une augmentation significative de la population, à un changement radical de société dont la hiérarchisation est perceptible avec l'aménagement des enceintes et de quelques grands bâtiments. À Carvin (Pas-de-Calais) [cf. encadré p 105], une vaste enceinte à plusieurs fossés et palissades renfermant un mobilier abondant caractéristique du Néolithique moyen II a été quasi intégralement fouillée. Elle ceinture deux bâtiments à poteaux centraux supportant une faîtière, qui sont accompagnés de quelques fosses. Cet agencement pose la question de la fonction même de ce type d'ouvrage monumental. Carvin est l'un des rares exemples où des bâtiments de ce type ont été 
découverts à l'intérieur même d'une enceinte. Dans ces structures, on met généralement au jour quelques fosses isolées, rarement plus. La raison principale en est l'érosion qui fait disparaître les structures les moins profondes et les difficultés importantes de reconnaissance du plan des architectures.

Les bâtiments sur poteaux centraux sont caractéristiques du Néolithique moyen II. On les trouve associés ou non à des enceintes. On constate qu'ils sont attestés sur une large portion du territoire français, au sud comme au nord de la Loire. Ces récentes découvertes permettent d'ores et déjà d'établir des parallèles entre ces habitats et ces architectures. [...]

\section{Les bâtiments du Michelsberg de Cuiry-lès-Chaudardes}

Caroline Colas

Inrap

L occupation Michelsberg se compose de quatre bâtiments accompagnés d'une sépulture et de quelques fosses [Fig.1] (Colas et al., à paraître). Trois autres bâtiments partiellement reconnus existent probablement. Les types de bâtiments, leur couverture, leur mobilier permettent de percevoir le caractère exceptionnel du site. La façade occidentale, composée de quatre (bât. 1, 2 et 4) ou six (bât. 3, 5 et 7 ?) trous de poteaux, forme une abside tandis que la façade orientale, plus étroite, n'en possède que deux [Fig.2]. Leur longueur, autour d'une dizaine de mètres de long, est stable tandis que la largeur varie de 3 et $6 \mathrm{~m}$, pour le petit côté, et de 4 et $8 \mathrm{~m}$, pour le grand. On peut ainsi distinguer deux catégories d'après leurs proportions : les bâtiments larges, dont la grande largeur correspond à environ la moitié de la longueur, et les bâtiments étroits, où elle n'en représente que le tiers.

Le diamètre des trous de poteaux oscille entre 0,15 et $0,80 \mathrm{~m}$ et leur profondeur entre $0,06 \mathrm{~m}$ (pour les plus arasés) et o,30 m (pour les mieux préservés). La plupart ont conservé l'empreinte du poteau disparu (fantôme). Il s'agit donc d'avant-trous destinés à recevoir le pilier en bois. La lecture des plans révèle l'absence de poteaux dans l'espace interne. L'espacement entre les poteaux latéraux suggère des murs extérieurs composés de poteaux d'armature destinés probablement à recevoir un clayonnage et du torchis et à supporter le toit. L'absence de poteaux faîtiers pose, cependant, problème si on envisage un toit à double pente. Des calculs effectués par des chercheurs allemands, en tenant compte des contraintes liées aux vents, au poids de la neige ainsi qu'au poids d'une couverture en motte de gazon, proposent deux possibilités pour couvrir un espace d'une dizaine de mètres (Zeeb-Lanz, 2001) : les chevrons du toit reposent sur les murs et sur le sol ou bien les chevrons sont enfoncés à l'oblique dans le sol [Fig.3]. La poutre faîtière est, dans ce cas, ligaturée au niveau de l'entrecroisement des chevrons. Donc, à moins de supposer l'existence de poteaux centraux ou d'une tranchée de fondation externe aujourd'hui disparus, la charpente devait reposer directement sur les murs extérieurs.

De même, l'espace interne n'était pas divisé ou bien les cloisons et les aménagements secondaires ont disparu. Le bâtiment 3 échappe à ce modèle. Plus large, il possède davantage de poteaux au niveau de l'abside. Une structure centrale composée de 4 poteaux était peut-être destinée à diminuer la portance et soutenir le toit [Fig.2]. Le bâtiment 5 appartient vraisemblablement à cette même famille. Le nombre de poteaux et la forme de l'abside conservée permettent un rapprochement avec le bâtiment 3. De même, la structure 142 a finalement été considérée sur plan comme un probable bâtiment supplémentaire, construit sur le même modèle que le bâtiment 3, mais dont les poteaux externes auraient disparu (orientation similaire). Des traces de structures en creux, considérées comme non probantes sur le terrain, pourraient en fait être le fond très mal conservé des poteaux de l'abside. Les absides, localisées du côté occidental, sont peut-être à interpréter comme des pignons d'entrée protégeant des pluies dominantes et des vents froids, comme c'est le cas pour les maisons du Néolithique ancien. Excepté cet argument indirect, rien, dans les plans, ne permet de situer précisément les entrées.

Les aspects techniques des tessons permettent de les assimiler à ceux de la culture Michelsberg (4. 200-3500 av. notre ère). Plusieurs trous de poteaux contenaient également des éclats de silex, de la faune et même un fragment de meule. La rareté des structures autour des unités d'habitation nous fait exclure la possibilité de mobilier erratique piégé lors du rebouchage des trous de poteaux. La présence de fragments d'os dans presque tous les bâtiments a permis d'effectuer des datations radiocarbones et de vérifier la pertinence $\mathrm{du}$ rattachement. Les cinq analyses situent cette occupation au Michelsberg récent entre 3700 et 3400 av. notre ère à deux sigmas Le seul autre gisement de cette période dans l'Aisne se situe à 800 mètres à l'ouest aux « Fontinettes ». Il s'agit du seul autre habitat du Michelsberg « ouvert ». Tous les autres sites sont des enceintes et un monument funéraire, et datent du Michelsberg ancien (4, 200-4 000 av. notre ère) à deux sigmas. Le seul autre site d'habitat ouvert de cette période dans l'Aisne se situe à 800 mètres à l'ouest aux «Fontinettes». Tous les autres sites sont des enceintes et un monument funéraire, datés du Michelsberg ancien (4, 200-4, 000 av. notre ère).

Outre le plan potentiel mal conservé d'un bâtiment aux «Fontinettes », les sites de Berryau-Bac et d'Osly-Courtil sont les seuls de la région à avoir fourni des plans de maisons. Le bâtiment de Berry-au-Bac "Le Vieux Tordoir" est proche de ceux de Cuiry par ses dimensions (14 m de long sur $4 \mathrm{~m}$ de large) mais il en différe par la présence d'un rangée centrale de poteaux et son orientation nord-est/sud-ouest. La présence ou non d'une abside 